JLAB-THY-00-26

\title{
Renormalons as dilatation modes in the functional space
}

\author{
A. BABANSKY and I. BALITSKY \\ Physics Department, Old Dominion University, Norfolk VA 23529 \\ and \\ Theory Group, Jefferson Lab, Newport News VA 23606 \\ e-mail: balitsky@jlab.org and babansky@jlab.org
}

\begin{abstract}
There are two sources of the factorial large-order behavior of a typical perturbative series. First, the number of the different Feynman diagrams may be large; second, there are abnormally large diagrams known as renormalons. It is well known that the large combinatorial number of diagrams is described by instanton-type solutions of the classical equations. We demonstrate that from the functional-integral viewpoint the renormalons do not correspond to a particular configuration but manifest themselves as dilatation modes in the functional space.
\end{abstract}

PACS numbers: 12.38.Cy, 11.15.Bt, 11.15.Kc 


\section{INTRODUCTION}

It is well known that the perturbative series in a typical quantum field theory is at best asymptotic: the coefficients in front of a typical perturbative expansion grow like $n$ ! where $\mathrm{n}$ is the order of the perturbation series (see e.g. the book [i] $\left.i_{1}\right)$. There are two sources of the $n$ ! behavior which correspond to two different situations. In the first case all Feynman diagrams are $\sim 1$ but their number is large $(\sim n !)$ [림] . In the second case, we have just one diagram but it is abnormally big $-\sim n$ ! (the famous 't Hooft renormalon [젬]). The first type of factorial behavior is not specific to a field theory; for example, it can be studied in the anharmonic-oscillator quantum mechanics. On the contrary, renormalon singularities can occur only in field theories with running coupling constant (for the review of the renormalons, see ref. $\left[\begin{array}{l}\overline{4} \\ 1\end{array}\right]$ and references therein).

It is convenient to visualize the large-order behavior of perturbative series using the 't Hooft picture of singularities [30ㄱ. Consider Adler's function related to the polarization operator in QCD

$$
\begin{aligned}
& D\left(q^{2}\right)=4 \pi^{2} q^{2} \frac{d}{d q^{2}} \frac{1}{3 q^{2}} \Pi\left(q^{2}\right) \\
& \Pi\left(q^{2}\right)=\int d x e^{i q x} \int D \bar{\psi} D \psi D A j_{\mu}(x) j_{\mu}(0) e^{-S_{\mathrm{QCD}}}
\end{aligned}
$$

Suppose we write down $D\left(q^{2}\right)$ as a Borel integral

$$
D\left(\alpha_{s}(q)\right)=\int_{0}^{\infty} d t D(t) e^{-\frac{4 \pi}{\alpha_{s}(q)} t}
$$

The divergent behavior of the original series $D\left(\alpha_{s}(q)\right.$ is encoded in the singularities of its Borel transform shown in Fig.1. In QCD, we have two types of singularities: renormalons

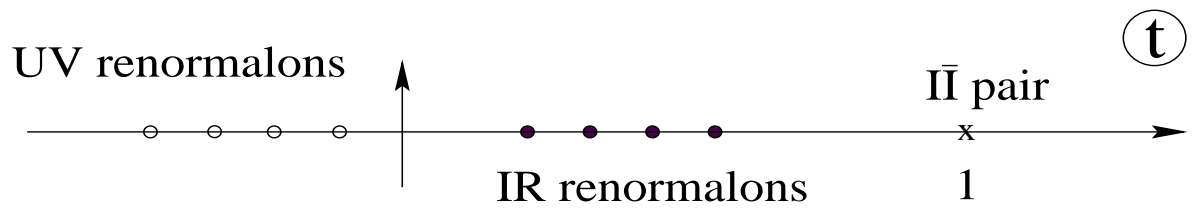

FIG. 1. 't Hooft picture of singularities in the Borel plane for QCD. 
(ultraviolet or infrared) and instanton-induced singularities ${ }_{L}^{\mathbb{F}_{1}}$ The ultraviolet (UV) renormalons are located at $t=-\frac{1}{b}, \frac{2}{b}, \frac{3}{b} \ldots\left(b=11-\frac{2}{3} n_{f}\right)$. In terms of Feynman diagrams they come from the regions of hard momenta in renormalon bubble chain, see Fig. 2. The infrared
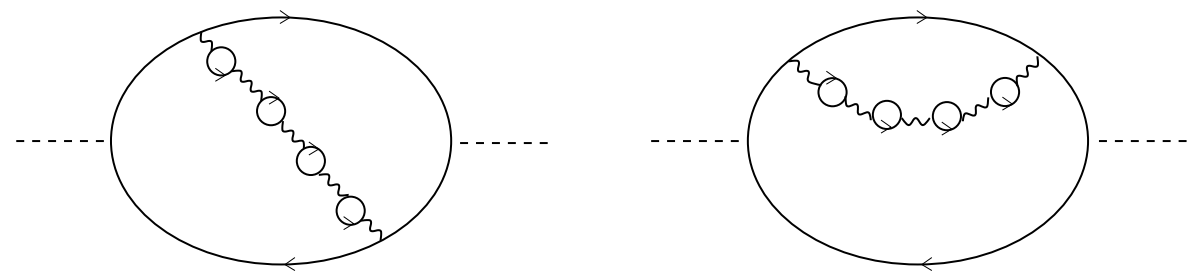

FIG. 2. Typical renormalon bubble chain diagrams for the polarization operator in QCD.

(IR) renormalons placed at $t=\frac{2}{b}, \frac{3}{b} \ldots$ come from the region of soft momenta in the bubble chain diagrams. The instanton singularities are located at $t=1,2,3, \ldots$ and they correspond to the large number of graphs which are summed up to the contribution of $I \bar{I}$ configurations in the functional space. The main result of this paper is that unlike the instanton-type singularities, the renormalons do not correspond to a particular configuration but manifest themselves as dilatation modes in the functional space.

The paper is organized as follows. In Section 2 we relate Borel representation to the functional integral along the instanton-antiinstanton valley in the double-well quantum mechanics. In Section 3 we describe the conformal valley in QCD and discuss the relation between valleys and Borel representation in the field theories with running coupling constant. Sect. 4 and 5 are devoted to the interpretation of IR and UV renormalons as dilatation modes of the valley. In the last section we discuss general aspects of our approach and outline the direction of the future work.

\footnotetext{
* Actually, the instanton itself is not related to the divergence of perturbative series since it belongs to a different topological sector. The first topologically trivial classical configuration, which contributes to the divergence of perturbation theory, is a weakly coupled instanton-antiinstanton

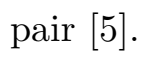




\section{VALLEYS AND BOREL PLANE IN QUANTUM MECHANICS}

The interpretation of renormalons as dilatation modes is based upon the similarity of the functional integral in the vicinity of a valley in the functional space [i, $[\bar{b}]$ to the Borel representation (2). At first, we will consider the quantum mechanical example without renormalons and then demonstrate that in a field theory the same integral along the valley leads to renormalon singularities. With QCD in view, we consider the double-well anharmonic oscillator described by the functional integral

$$
Z=\int D \phi e^{-\frac{S(\phi)}{g^{2}}}
$$

where

$$
S(\phi)=\int d t\left(\frac{\dot{\phi}^{2}}{2}+\frac{\left.\phi^{2}(1-\phi)^{2}\right)}{2}\right)
$$

The large-order behavior in this model is determined by the instanton-antiinstanton $(I \bar{I})$ configuration. The $I \bar{I}$ valley for the double-well system may be chosen as

$$
f_{\alpha}(t-\tau)=\frac{1}{2} \tanh \frac{t-\tau+\alpha}{2}-\frac{1}{2} \tanh \frac{t-\tau-\alpha}{2}
$$

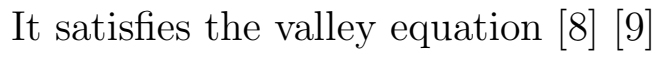

$$
\frac{12}{\xi^{2}} w_{\alpha}(t) f_{\alpha}^{\prime}(t)=L_{\alpha}(t)
$$

where $\xi \equiv e^{\alpha}, f_{\alpha}^{\prime} \equiv \frac{\partial}{\partial \alpha} f_{\alpha}$, and $L_{\alpha}(t)=\left.\frac{\delta S}{\delta \phi}\right|_{\phi=f_{\alpha}(t)}$. Here $w_{\alpha}(t)=\frac{\xi}{4} \sinh \alpha(\cosh \alpha \cosh t+1)^{-1}$

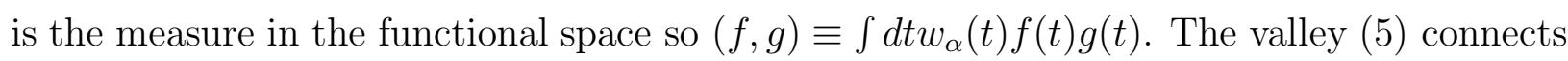
two classical solutions: the perturbative vacuum at $\alpha=0$ and the infinitely separated $I \bar{I}$ pair at $\alpha \rightarrow \infty$. The $I \bar{I}$ separation $\alpha$ and the position of the $I \bar{I}$ pair $\tau$ are the two collective coordinates of the valley. In order to integrate over the small fluctuations near the configuration ( $(\underline{5})$, we introduce two $\delta$-functions $\delta\left(\phi(t)-f_{\alpha}(t-\tau), \dot{f}_{\alpha}(t-\tau)\right)$ and $\delta(\phi(t)-$ $\left.f_{\alpha}(t-\tau), f_{\alpha}^{\prime}(t-\tau)\right)$ restricting the integration along the two collective coordinates. Following the standard procedure (Faddeev-Popov trick), we insert 


$$
1=\int d \alpha\left[\left(f_{\alpha}^{\prime}, f_{\alpha}^{\prime}\right)+\left(\phi-f_{\alpha}, f_{\alpha}^{\prime}+\frac{w^{\prime}}{w} f_{\alpha}\right)\right] \delta\left(\phi-f_{\alpha}, f_{\alpha}^{\prime}\right)
$$

and

$$
1=\int d \tau\left[\left(\dot{f}_{\alpha}, \dot{f}_{\alpha}\right)+\left(\phi-f_{\alpha}, \dot{f}_{\alpha}+\frac{\dot{w}}{w} f_{\alpha}\right)\right] \delta\left(\phi-f_{\alpha}, \dot{f}_{\alpha}\right)
$$

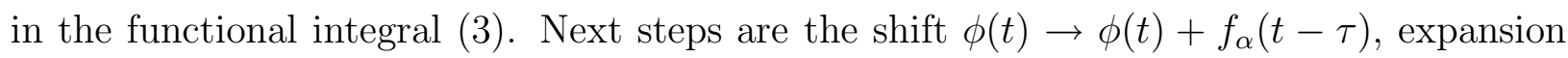
in quantum deviations $\phi(t)$ and the gaussian integration in the first nontrivial order in perturbation theory. After the shift, the the linear term in the exponent $\int d t \phi(t) L_{\alpha}(t)$ is

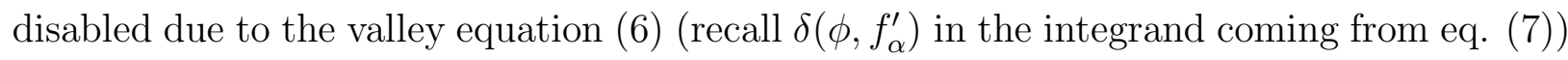
so the functional integral $\left(\begin{array}{c}\overline{1} \\ \mathbf{N}_{1}\end{array}\right)$ for the vacuum energy in the leading order in $g^{2}$ reduces to

$$
T \int d \alpha \int D \phi\left(f_{\alpha}^{\prime}, f_{\alpha}^{\prime}\right)\left(\dot{f}_{\alpha}, \dot{f}_{\alpha}\right) \delta\left(\phi, f_{\alpha}^{\prime}\right) \delta\left(\phi, \dot{f}_{\alpha}\right) e^{-\frac{1}{g^{2}}\left[S_{\alpha}+\frac{1}{2} \int d t \phi(t) \square_{\alpha} \phi(t)\right]}+O\left(g^{2}\right)
$$

Here T (the total "volume" in one space-time dimension) is the result of trivial integration over $\tau, \square_{\alpha}=-\partial^{2}+1-6 f_{\alpha}\left(1-f_{\alpha}\right)$ is the operator of second derivative of the action and

$$
S_{\alpha} \equiv S(\xi)=\frac{6 \xi^{4}-14 \xi^{2}}{\left(\xi^{2}-1\right)^{2}}-\frac{17}{3}+\frac{12 \xi^{2}+4}{\left(\xi^{2}-1\right)^{3}} \ln \xi
$$

is the action of $I \bar{I}$ valley. Performing Gaussian integrations we get

$$
\begin{aligned}
& T \frac{1}{g^{2}} \int_{0}^{\infty} d \alpha e^{-\frac{1}{g^{2}} S_{\alpha}} F(\alpha), \\
& F(\alpha)=\frac{\left(\operatorname{det} \square_{\alpha}\right)^{-1 / 2}\left(f_{\alpha}^{\prime}, f_{\alpha}^{\prime}\right)\left(\dot{f}_{\alpha}, \dot{f}_{\alpha}\right)}{\left(f_{\alpha}^{\prime}, \square_{\alpha}^{-1} w_{\alpha} f_{\alpha}^{\prime}\right)^{1 / 2}\left(\dot{f}_{\alpha}, \square_{\alpha}^{-1} w_{\alpha} \dot{f}_{\alpha}\right)^{1 / 2}} .
\end{aligned}
$$

At $\alpha \rightarrow \infty$ we have the widely separated $I$ and $\bar{I}$. In this case, the determinant of the $I \bar{I}$ configuration factorizes into a product of two one-instanton determinants (with zero modes excluded) so that $F(\alpha) \rightarrow$ const at $\alpha \rightarrow \infty$. The divergent part of the integral ('ili) corresponds to the second iteration of the one-instanton contribution to the vacuum energy, therefore it must be subtracted from the $I \bar{I}$ contribution to $E_{\mathrm{vac}}$ :

$$
g^{2} E_{\mathrm{vac}}\left(g^{2}\right)=\int d \alpha\left(e^{-\frac{1}{g^{2}} S_{\alpha}} F(\alpha)-e^{-\frac{1}{g^{2}} 2 S_{I}} F(\infty)\right)
$$

where $S_{I}$ is the one-instanton action. Since $S_{\alpha}$ is a monotonous function of $\alpha$ one can invert Eq. ('īioj) and obtain 


$$
g^{2} E_{\mathrm{vac}}\left(g^{2}\right)=\int_{0}^{2 S_{I}} d S e^{-\frac{1}{g^{2}} S} F(S)\left(\frac{1}{S-2 S_{I}}\right)_{+}
$$

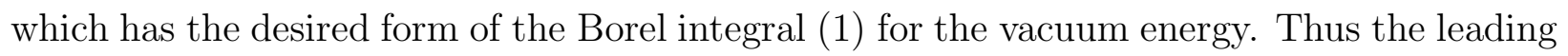
singularity for $E_{\mathrm{vac}}(S)$ is $F\left(2 S_{I}\right) /\left(S-2 S_{I}\right)$. Note that our semiclassical calculation does not give the whole answer for the Borel transform of vacuum energy $\left(F(S) \neq E_{\mathrm{vac}}(S)\right.$ in general) since we threw away the higher quantum fluctuations around the $I \bar{I}$ pair in Eq. (6i) yet it determines the the leading singularity in the Borel plane.

\section{III. $I \bar{I}$ VALLEY AND BOREL INTEGRAL IN QCD}

The situation with the instanton-induced asymptotics of perturbative series in a field theory such as QCD is pretty much similar to the case of quantum mechanics with one notable exception: in QCD there is an additional dimensional parameter - the overall size of the $I \bar{I}$ configuration $\rho$. The classical $I \bar{I}$ action does not depend on this parameter but the quantum determinant does, leading to the replacement

$$
e^{-\frac{1}{g^{2}} S} \rightarrow e^{-\frac{1}{g^{2}(\rho)} S}
$$

so the Borel integrand have the following generic form

$$
F(S) \sim \int d \rho e^{-\frac{1}{g^{2}(\rho)} S} \mathcal{F}(\rho)
$$

The divergence of this integral at either large or small $\rho$ determines the position of singularities of $F(s)$. We will demonstrate (by purely dimensional analysis) that $\mathcal{F}(\rho) \sim \rho^{-5}$ at

$\rho \rightarrow \infty$ and $\mathcal{F}(\rho) \sim \rho$ at $\rho \rightarrow 0$ leading to the IR renormalon at $S=\frac{32 \pi^{2}}{b}$ and the UV renormalon at $S=-\frac{16 \pi^{2}}{b}$, respectively 䨤.

\footnotetext{
$\dagger$ 'In its present form, our analysis of renormalons is applicable to the "off-shell" processes which can be related to the Euclidean correlation functions of two (or more) currents. An example of a different type is the IR renormalon in the pole mass of a heavy quark located at $S=\frac{16 \pi^{2}}{b}[\bar{i} \mathbf{I}]$
} 


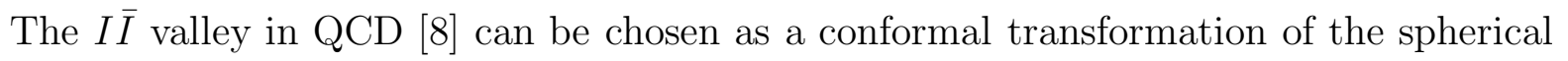
configuration

$$
A_{\mu}(x)=-i \frac{\bar{\sigma}_{\mu} x-x_{\mu}}{x^{2}} f_{\alpha}(t)
$$

with $t=\ln x^{2} / d^{2}$ where $d$ is an arbitrary scale. (We use the notations $x \equiv x_{\mu} \sigma_{\mu}, \quad \bar{x} \equiv x_{\mu} \bar{\sigma}_{\mu}$ where $\left.\sigma_{\mu}=(1,-i \vec{\sigma}), \quad \bar{\sigma}_{\mu}=(1, i \vec{\sigma})\right)$. To obtain the $I \bar{I}$ configuration with arbitrary sizes $\rho_{1}, \rho_{2}$ and separation $R$ one performs shift $x \rightarrow x-a$, inversion $x \rightarrow \frac{d^{2}}{x^{2}} x$ and second shift $x \rightarrow x-x_{0}$ (see Fig. 3). The resulting valley is the sum of the $I$ and $\bar{I}$ in the singular gauge
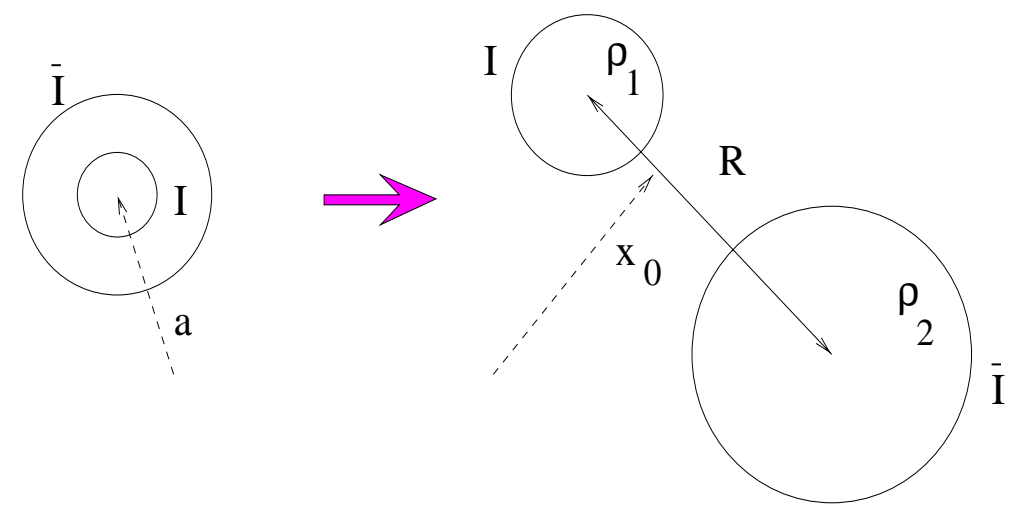

FIG. 3. Conformal $I \bar{I}$ valley in QCD.

in the maximum attractive orientation plus the additional term (which is small at large $I \bar{I}$ separations)

$$
A_{\mu}^{v}(x)=A_{\mu}^{I}\left(x-x_{0}\right)+A_{\mu}^{\bar{I}}\left(x-x_{0}\right)+B_{\mu}\left(x-x_{0}\right)
$$

where

$$
\begin{aligned}
& A_{\mu}^{I}(x)=-i \rho_{1}^{2} \frac{\sigma_{\mu} \bar{x}-x_{\mu}}{x^{2}\left(x^{2}+\rho_{1}^{2}\right)} \\
& A_{\mu}^{\bar{I}}(x)=-i \rho_{2}^{2} \frac{R \bar{\sigma}_{\mu}(x-R) \bar{R}-(x-R)_{\mu}}{R^{2}(x-R)^{2}\left((x-R)^{2}+\rho_{2}^{2}\right)} .
\end{aligned}
$$

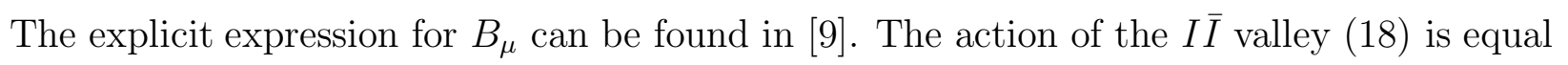

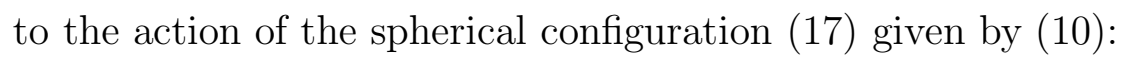

$$
S^{v}(z)=48 \pi^{2} S(\xi), \quad \xi=z+\sqrt{z^{2}-1},
$$


where the "conformal parameter" $z$ is given by

$$
z=\left(\rho_{1}^{2}+\rho_{2}^{2}+R^{2}\right) /\left(2 \rho_{1} \rho_{2}\right)
$$

Let us now calculate the polarization operator (1in in the valley background. The collective coordinates are the sizes of instantons $\rho_{i}$, separation $R$, overall position $x_{0}$ and the orientation in the color space (the valley of a general color orientation has the form $\mathcal{O}_{a b} A_{b}^{v}$ where $\mathcal{O}$ is an arbitrary $S U(3)$ matrix). The structure of Gaussian result for the polarization operator in the valley background is

$$
\int_{0}^{\infty} \frac{d \rho_{1} d \rho_{2}}{\rho_{1}^{5} \rho_{2}^{5}} d^{4} R d^{4} x_{0} d \mathcal{O} \Pi^{v}(q) \frac{1}{g^{17}} e^{-\frac{S^{v}(z)}{g^{2}}} \Delta\left(\rho_{i}, R\right)
$$

where 17 is a number of the collective coordinates Here $\Pi^{v}(q)$ is a Fourier transform of

$$
\Pi^{v}(x)=\left(\sum e_{q}^{2}\right) \operatorname{Tr} \gamma_{\mu} G(x, 0) \gamma_{\mu} G(0, x)
$$

where $G(x, y)$ is the Green function in the valley background. The factor $\Delta\left(\rho_{i}, R\right)$ in Eq. $(\overline{2} \overline{2} \overline{2})$ is the quantum determinant - the result of Gaussian integrations near the valley (1' $\left.\overline{1}_{1} \overline{8}_{1}\right)$ with the additional factors due to the restricted integrations along the collective coordinates (cf. eq. (1) 121$)$ ). For our purposes, it is convenient to introduce the conformal parameter $z$ and the average size $\rho=\sqrt{\rho_{1} \rho_{2}}$ as the collective coordinates in place of $\rho_{1}$ and $\rho_{2}$. We have

$$
\int d z \frac{d \rho}{\rho^{9}} d^{4} R d^{4} x_{0} \Pi^{v}(q) \frac{1}{g^{17}(\rho)} F\left(z, R^{2} / \rho^{2}\right) e^{-\frac{S^{v}(z)}{g^{2}(\rho)}}
$$

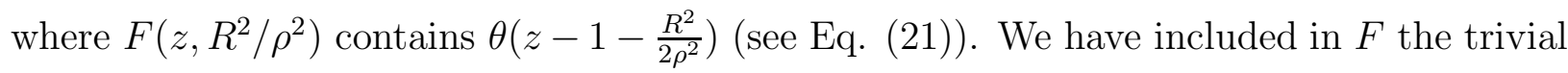
integral over color orientation which gives the volume of $S U(3)$ group.

The main effect of the quantum determinant $\Delta$ is the replacement of the bare coupling constant $g^{2}$ in Eq. (2) by the effective coupling constant $g^{2}(\rho)$ so the remaining function $F$ is

\footnotetext{
${ }^{\ddagger}$ At $z \rightarrow \infty$ (for weakly interacting $I$ and $\bar{I}$ ) the mode corresponding to relative orientation in the color space becomes non-gaussian so one should introduce an additional collective coordinate corresponding to this quasizero mode. We are interested, however, in $z \sim 1$ where this mode is still Gaussian so it is taken into account in quantum determinant $\Delta$.
} 
the (dimensionless) function of the ratio $R^{2} / \rho^{2}$ and the conformal parameter. This is almost evident from the renormalizability of the theory since the only dimensional parameters are $\rho$ and $R$. Formally, one can prove that rescaling of the configuration by factor $\lambda$ (so that $\rho \rightarrow \lambda \rho$ and $R \rightarrow \lambda R$ ) leads at the one-loop level to multiplication of the determinant by a factor $\lambda^{b S^{v}(z) / 8 \pi^{2}}$ due to conformal anomaly (see Appendix).

\section{IR RENORMALON FROM THE DILATATION MODE OF THE $I \bar{I}$ VALLEY}

Consider the singularities of the integral $(\overline{2} \overline{2} \overline{3})$. The function $F\left(z, R^{2} / \rho^{2}\right)$ is non-singular since the singularity in $\Phi$ (三 singularity in $\Delta)$ would mean a non-existing zero mode in quantum determinant. Moreover, the integration over $R$ is finite due to $\theta\left(z-1-\frac{R^{2}}{2 \rho^{2}}\right)$ indicating that the only source of singularity at finite $z$ is the divergence of the $\rho$ integral at either large or small $\rho$. (At $z \rightarrow \infty$, in a way similar to the derivation of Eq. (1) (1) we obtain the first instanton-type singularity located at $t=1[1][0][]]$.

Let us demonstrate that the singularity of the integral (i2 $(2 \bar{i})$ at large $\rho \gg \frac{1}{q}$ corresponds to the IR renormalon. The polarization operator $\Pi(q)$ in the background of the large-scale vacuum fluctuation reduces to [i] $\left.\mathbf{1}_{1}^{1} \mathbf{1}_{1}\right]$ (see Fig. 3)

$$
\Pi(q)=\int d^{4} x e^{i q x}\left\langle j_{\mu}(x) j_{\mu}(0)\right\rangle_{A} \rightarrow-\frac{\sum e_{q}^{2}}{16 \pi^{2}}\left(\frac{G^{2}(0)}{q^{2}}+c \alpha_{s} \frac{G^{3}(0)}{q^{4}}+\ldots\right)
$$

where $G^{2} \equiv 2 \operatorname{Tr} G_{\xi \eta} G_{\xi \eta}, G^{3} \equiv 2 \operatorname{Tr} G_{\xi \eta} G_{\eta \sigma} G_{\sigma \xi}$, and $c$ is an (unknown) constant. (The coefficient in front of $G^{3}$ vanishes at the tree level [1] $\left.\left.\overline{1}_{1}\right]\right)$. Consider the leading term in this
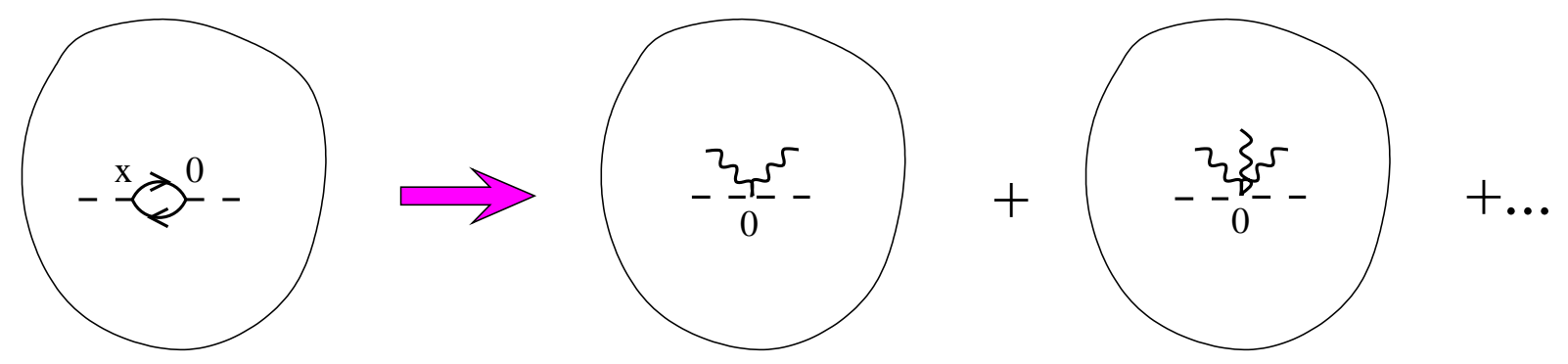

FIG. 4. Expansion of the polarization operator in large-scale external fields.

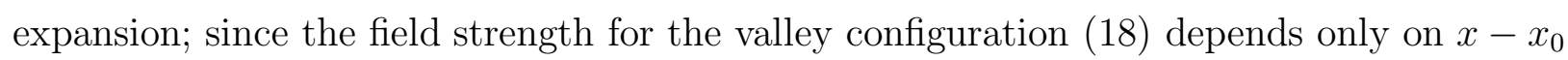




$$
\int d^{4} x_{0} \operatorname{Tr} G_{\xi \eta}^{v}(0) G_{\xi \eta}^{v}(0)=4 S^{v}(z)
$$

the intergal (플

$$
\frac{1}{q^{2}} \int_{1}^{\infty} d z \int_{0}^{\infty} \frac{d \rho}{\rho^{9}} d^{4} R \frac{1}{g^{17}(\rho)} F\left(z, R^{2} / \rho^{2}\right) e^{-\frac{S^{v}(z)}{g^{2}(\rho)}}
$$

where we have included the factor $\frac{1}{4 \pi^{2}} \sum e_{q}^{2} S^{v}(z)$ in $F$. The (finite) integration over $R$ can be performed resulting in an additional dimensional factor $\rho^{4}$ :

$$
\int d^{4} R F(\rho, z, R)=\rho^{4} \Phi(z)
$$

where the function $\Phi$ is dimensionless so it can depend only on $z$. Finally, we get

$$
\frac{1}{q^{2}} \int_{1}^{\infty} d z \int_{0}^{\infty} \frac{d \rho}{\rho^{5}} \frac{1}{g^{17}(\rho)} \Phi(z) e^{-\frac{1}{g^{2}(\rho)} S^{v}(z)} .
$$

Inverting Eq. (i[0]), we can write the corresponding contribution to Adler's function as an integral over the valley action $\left(t \equiv \frac{S}{16 \pi^{2}}\right)$ :

$$
D\left(q^{2}\right) \simeq \frac{1}{3 q^{4}} \int_{0}^{1} d t \int_{0}^{\infty} \frac{d \rho}{\rho^{5}} \frac{1}{g^{17}(\rho)} \Phi(t) e^{-\frac{4 \pi}{\alpha_{s}(\rho)} t} .
$$

Note that extra $\frac{1}{g^{2}(\rho)}$ in the numerator can be eliminated using integration by parts:

$$
\frac{16 \pi^{2}}{g^{2}(\rho)} \int_{0}^{1} d t e^{-\frac{4 \pi}{\alpha_{s}(\rho)} t} \Phi(t)=\int_{0}^{1} d t \Phi^{\prime}(t) e^{-\frac{4 \pi}{\alpha_{s}(\rho)} t}-\Phi(1) e^{-\frac{4 \pi}{\alpha_{s}(\rho)}}+\Phi(0) .
$$

The last two terms are irrelevant for the would-be renormalon singularity at $t=\frac{2}{b}$ (the term $\sim e^{-\frac{4 \pi}{\alpha_{s}(\rho)}}$ corresponds to the $I \bar{I}$ singularity and the term $\sim \Phi(0)$ does not depend on coupling constant). Likewise, extra $g(\rho)$ can be absorbed by Laplace transformation

$$
g(\rho) \int_{0}^{1} d t \Phi(t) e^{-\frac{4 \pi}{\alpha_{s}(\rho)} t}=4 \sqrt{\pi} \int_{0}^{1} d t e^{-\frac{4 \pi}{\alpha_{s}(\rho)} t} \int_{0}^{t} d t^{\prime}\left(t-t^{\prime}\right)^{-1 / 2} \Phi\left(t^{\prime}\right)
$$

leading to

$$
D\left(q^{2}\right)=\frac{1}{q^{4}} \int_{0}^{1} d t \int_{0}^{\infty} \frac{d \rho}{\rho^{5}} e^{-\frac{4 \pi}{\alpha_{s}(\rho)} t} \Psi(t)
$$

where $\Psi(t)=\frac{1}{3 \sqrt{\pi}(4 \pi)^{17}} \int_{0}^{t} d t^{\prime}\left(t-t^{\prime}\right)^{-1 / 2} \Phi^{(9)}\left(t^{\prime}\right)$ after nine integrations by parts and a Laplace transformation. Using the two-loop formula for $\alpha_{s}$ we get 


$$
D(t) \simeq \Psi(t) \int_{0}^{\infty} \frac{d \rho}{q^{4} \rho^{5}}\left(q^{2} \rho^{2}\right)^{b t}\left(\alpha_{s}(\rho) / \alpha_{s}(q)\right)^{\frac{2 b^{\prime} t}{b}}
$$

where $b^{\prime}=51-\frac{19}{3} n_{f}$. It is clear now that the integral over $\rho$ diverges in the IR region starting from $t=\frac{2}{b} \frac{1}{b}$ leading to in a singularity in $D(t)$ at $t=\frac{2}{b}$ This singularity is the first IR renormalon. At the one-loop level we can drop the ratio $\left(\alpha_{s}(\rho) / \alpha_{s}(q)\right)^{\frac{2 b^{\prime} t}{b}}$ so the renormalon is a simple pole $\frac{1}{t-2 / b}$. To get he character of this singularity at the two-loop level, we recall that an extra $\alpha(\rho)$ factor does not affect the singularity while an extra $\frac{1}{\alpha(q)}$ factor changes it from $\left(t-\frac{2}{b}\right)^{\lambda}$ to $\left(t-\frac{2}{b}\right)^{\lambda-1}$, as one can easily see from the integration by parts

$$
\frac{16 \pi^{2}}{g^{2}(q)} \int_{0}^{1} d t e^{-\frac{4 \pi}{\alpha_{s}(q)} t}\left(t-\frac{2}{b}\right)^{\lambda}=\int_{0}^{1} d t \lambda\left(t-\frac{2}{b}\right)^{\lambda-1} e^{-\frac{4 \pi}{\alpha_{s}(q)} t}+\ldots
$$

Therefore, the extra factor $\left(\alpha_{s}(\rho) / \alpha_{s}(q)\right)^{\frac{2 b^{\prime} t}{b}}$ will convert the simple pole $\frac{1}{t-2 / b}$ into a branching point singularity $\left(t-\frac{2}{b}\right)^{-1-4 \frac{b^{\prime}}{b^{2}}}\left[\begin{array}{l}1 \\ 1\end{array} \overline{2}\right]$.

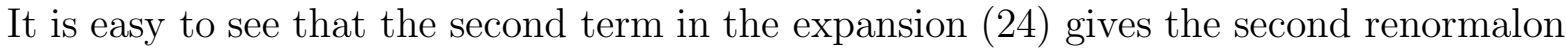
singularity located at $t=\frac{3}{b}$. Higher terms of the expansion of the polarization operator ( $\left.24 \overline{4}\right)$ will give the subsequent IR renormalons located at $t=\frac{4}{b}, \frac{5}{b}, \frac{6}{b}$ etc.

\section{UV RENORMALON AS A DILATATION MODE}

Next we demonstrate that the divergence of the integral $\left(\overline{2} \overline{3}_{-1}^{\prime}\right)$ at small $\rho$ leads to the UV renormalon. In order to find the polarization operator in the background of a very small

$\S$ Strictly speaking, at finite $q$ we cannot get to the singularity since our semiclassical approach is valid up to $\rho<\Lambda_{Q C D}$ which translates to $\frac{2}{b}-t>\frac{1}{\ln q \Lambda_{Q C D}}$; therefore we must take the limit $q^{2} \rightarrow \infty$ as well.

** To avoid confusion, note that in proving that extra $\frac{1}{\alpha_{s}(\rho)}$ does not change the singularity, we used the fact that before the $\rho$ integration the function $\Phi(t)$, which is constructed from valley determinants, can be singular only at $t \rightarrow 1$ where these determinants acquire zero modes. 
vacuum fluctuation (1) (1)

$$
A_{\mu}^{v}\left(x-x_{0}\right)=\frac{\rho^{2}}{\left(x-x_{0}\right)^{2}}\left(\delta_{\mu \alpha}-2 \frac{\left(x-x_{0}\right)_{\mu}\left(x-x_{0}\right)_{\alpha}}{y^{2}}\right) A_{\alpha}^{s}\left(\frac{\rho^{2}}{\left(x-x_{0}\right)^{2}}\left(x-x_{0}\right)\right)
$$

of the spherical configuration (1; $\left.\bar{Z}_{1}^{1}\right)$ (gauge rotated by $x / \sqrt{x^{2}}$ )

$$
\begin{aligned}
& A_{\alpha}^{s}(x)= \\
& -i\left[\sigma_{\alpha}(\bar{x}-\bar{a})-(x-a)_{\alpha}\right]\left(\frac{1}{(x-a)^{2}+\rho^{2} \xi}+\frac{\rho^{2} / \xi}{(x-a)^{2}\left((x-a)^{2}+\rho^{2} / \xi\right.}\right)
\end{aligned}
$$

where $a=R(\xi-1 / \xi)$ (we chose $d=\rho$ for the inversion). The corresponding transformation of the polarization operator has the form

$$
\Pi^{v}(x)=\left(\delta_{\mu \alpha}-2 \frac{y_{\mu} y_{\alpha}}{y^{2}}\right)\left\langle j_{\alpha}\left(\frac{\rho^{2}}{y^{2}} y\right) j_{\beta}\left(-\frac{\rho^{2}}{x_{0}^{2}} x_{0}\right)\right\rangle_{A_{s}}\left(\delta_{\beta \mu}-2 \frac{x_{0 \beta} x_{0 \mu}}{x_{0}^{2}}\right)
$$

where we use the notation $y \equiv x-x_{0}$. In the limit $\rho \rightarrow 0$ we need the polarization operator in the background of $A^{s}$ at small distances (see Fig. 4), so we can use the expansion ( $\left(2 \overline{2} \bar{u}^{\prime}\right)$
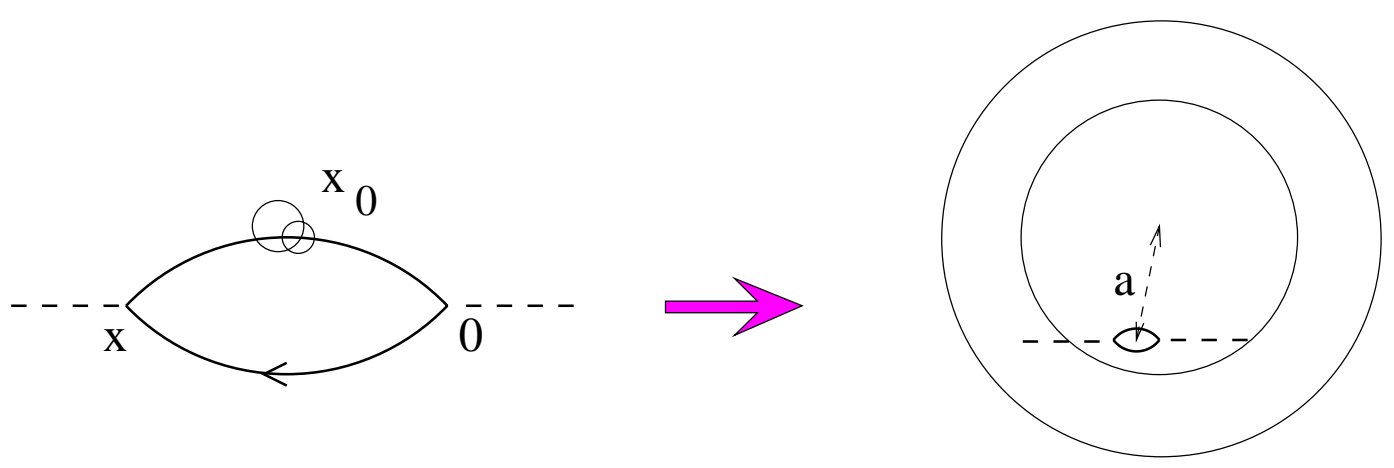

FIG. 5. Inversion of the polarization operator in the valley background.

(in the coordinate space)

$$
\left\langle j_{\mu}(x) j_{\nu}(0)\right\rangle_{A} \rightarrow-\frac{\sum e_{q}^{2}}{384 \pi^{4}}\left[\frac{2 x_{\mu} x_{\nu}+x^{2} \delta_{\mu \nu}}{x^{4}} G^{2}(0)+c \alpha_{s} G^{3}(0)\left(2 \frac{x_{\mu} x_{\nu}}{x^{2}}-3 \delta_{\mu \nu} \ln x^{2}\right)+\ldots\right]
$$

and obtain

\footnotetext{
${ }^{\dagger}$ To get the Eq. (1' $\left.1 \overline{8}_{1}^{\prime}\right)$ literally, this inversion must be accompanied by the gauge rotation with the matrix $x(x-R) R / \sqrt{x^{2}(x-R)^{2} R^{2}}$, see ref. [품]
} 


$$
\begin{aligned}
& \Pi^{v}(x) \rightarrow \\
& \frac{\sum e_{q}^{2}}{192 \pi^{4}}\left[\frac{\rho^{8} G_{s}^{2}(0)}{y^{4} x_{0}^{4} x^{2}}\left\{1-\frac{4\left(x_{0} y\right)^{2}}{x_{0}^{2} y^{2}}\right\}+c \alpha_{s} \frac{\rho^{12} G_{s}^{3}(0)}{y^{6} x_{0}^{6}}\left\{\left(3 \ln \frac{\rho^{2} x^{2}}{y^{2} x_{0}^{2}}-1\right) \frac{2\left(x_{0} y\right)^{2}}{x_{0}^{2} y^{2}}+1\right\}\right]
\end{aligned}
$$

where $G_{\mu \nu}^{s}(0)$ is the field strength of the spherical configuration (13.5. origin. After the integration over $x$ and $x_{0}$ the first term $\sim G_{s}^{2}$ vanishes and the second gives

$$
\int d x e^{i q x} \int d x_{0} \frac{1}{y^{6} x_{0}^{6}}\left[\left(3 \ln \frac{x^{2}}{y^{2} x_{0}^{2}}-1\right) \frac{2\left(x_{0} y\right)^{2}}{x_{0}^{2} y^{2}}+1\right]=\frac{\pi^{4}}{32}(6 \ln \pi+3 C-2) q^{4} \ln ^{2} q^{2},
$$

leading to

$$
\int d x d x_{0} e^{i q x} \Pi^{v}(x)=c^{\prime} \alpha_{s}(q) \frac{\sum e_{q}^{2}}{64} \rho^{6} \mathcal{G}\left(z, R^{2} / \rho^{2}\right) q^{4} \ln ^{2} q^{2}
$$

where

$$
\mathcal{G}\left(z, R^{2} / \rho^{2}\right)=\rho^{6} G_{s}^{3}(0)
$$

is a dimensionless non-singular function of $z$ and $R^{2} / \rho^{2}$ (the explicit expression can be easily found from Eq. ( momenta in the loop with one extra gluon in the valley background. After inversion, the characteristic distances in the loop diagram determining the coefficient in front of $G_{s}^{3}$ are $\sim \rho^{2} \sqrt{\frac{x^{2}}{x_{0}^{2} y^{2}}}$ which means that before inversion the characteristic momenta in the loop were $\sim q$ (in the integral ( $\left.(\underline{\overline{4}} \overline{\mathbf{4}}) x^{2} \sim x_{0}^{2} \sim y^{2} \sim q^{-2}\right)$.

Performing the integration over $R$ we obtain the analog of the Eq. (13ii) for the UV renormalon

$$
D\left(q^{2}\right) \simeq q^{2} \alpha_{s}(q) \int_{0}^{1} d t \int_{0}^{\infty} d \rho \rho\left(\ln q^{2} \rho^{2}\right)^{2} e^{-\frac{4 \pi}{\alpha_{s}(\rho)} t} \tilde{\Psi}(t)
$$

leading to

$$
D(t) \simeq \tilde{\Psi}(t) q^{2} \int_{0}^{\infty} d \rho^{2}\left(q^{2} \rho^{2}\right)^{b t}\left(\ln q^{2} \rho^{2}\right)\left(\alpha_{s}(\rho) / \alpha_{s}(q)\right)^{\frac{2 b^{\prime} t}{b}}
$$

Note that extra $\alpha_{s}(q)$ in Eq. (1 124$)$ is compensated by one power of $\ln q^{2} \rho^{2}$. The integral over $\rho$ diverges at $t=-\frac{1}{b}$ which is the position of the first UV renormalon. At the oneloop level this renormalon is a double pole $(1+t / b)^{-2}$, same as in the perturbative analysis 


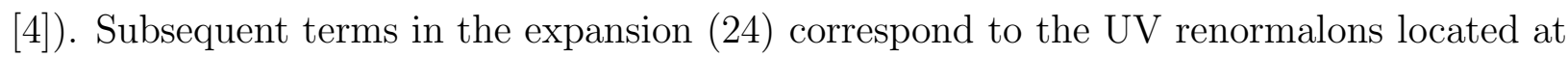
$t=-\frac{2}{b},-\frac{3}{b},-\frac{4}{b} \ldots$. It should be mentioned that the Eq. ('43i') cannot reproduce the strength

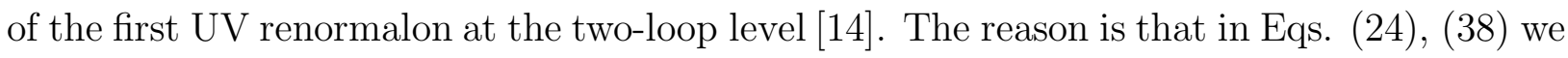
have neglected the anomalous dimensions of the operators $\sim\left(\alpha_{s}(q) / \alpha_{s}(\rho)\right)^{\frac{\gamma}{b}}$. Such factors can change the strength of the singularity. For the IR renormalon this does not matter since the operator $G^{2}$ is renorm-invariant $(\gamma=0)$ and for the subsequent renormalons we can easily correct our results by corresponding $\gamma$ 's. Unfortunately, for the UV renormalons we do not know how to use the conformal invariance with the anomalous dimensions included.

\section{CONCLUSIONS}

We have demonstrated that the integration along the dilatation modes in the functional space near the $I \bar{I}$ configuration leads to the renormalon singularities in the Borel plane. It is very important to note that we actually never use the explicit form of the valley configuration. What we have really used are the three facts: (I) conformal anomaly $\Rightarrow$ the fact that the rescaling of the vacuum fluctuation with an action S by a factor $\lambda$ multiplies

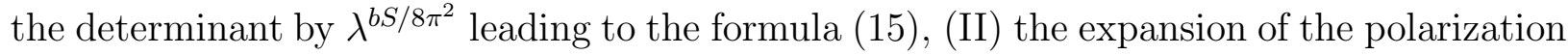
operator ( $\left(\overline{2}_{2}^{2}\right)$ in slow varying fields, and (III) the conformal invariance of QCD at the tree level (for the UV renormalon we wrote down the small-size configuration as an inversion of a large-scale vacuum fluctuation). All of these properties hold true for an arbitrary vacuum fluctuation so we could take an arbitrary valley and arrive at the same results Eq. (3isi) and

\footnotetext{
$\ddagger \ddagger$ We do not see the singularity at $t=\frac{1}{b}$ which is proposed in [1] due to the small-size monopoles which are beyond the scope of this paper (our results are based on the gaussian integration near the finite-action vacuum fluctuations while monopoles have infinite action).
} 
Eq. (4) dilatation mode in the functional space is general.

The form of the polarization operator in a valley background suggests the following parametrization of Adler' function as a double integral in $S$ and $\rho$

$$
D\left(\alpha_{s}(q)\right)=\int_{0}^{\infty} d t \int \frac{d \rho^{2}}{\rho^{2}} d\left(q^{2} \rho^{2}, t\right) e^{-\frac{4 \pi}{\alpha_{s}(\rho)} t}
$$

where

$$
d\left(q^{2} \rho^{2}, t\right) \sim\left(q^{2} \rho^{2}\right)^{-2} \text { at } \rho \rightarrow \infty, \quad d\left(q^{2} \rho^{2}, t\right) \sim\left(q^{2} \rho^{2}\right) \text { at } \rho \rightarrow 0
$$

The function $d\left(q^{2} \rho^{2}, t\right)$ contains only instanton-induced singularities in $t$ whereas the IR and UV renormalons come from the divergence of the integral (편) at large or small $\rho$, respectively. If we "switch off" the running coupling constant (e.g consider the conformal theory without $\beta$-function) the representation of Adler's function takes the form

$$
D^{\operatorname{conf}}\left(\alpha_{s}\right)=\int_{0}^{\infty} d t e^{-\frac{4 \pi}{\alpha_{s}} t} D^{\operatorname{conf}}(t)
$$

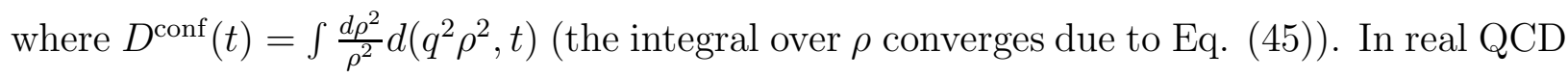
the function $D^{\text {conf }}\left(\alpha_{s}\right)$ defines the "conformal expansion" of the Adler's function with the coefficients coming from the "skeleton diagrams" in terms of usual perturbation theory [i] $\overline{1}_{\mathbf{6}}$.

As we mentioned above, our analysis of renormalons is applicable to the "off-shell" processes which can be related to the Euclidean correlation functions of two (or more) currents. One of possible directions of the future work would be to generalize these ideas to the renormalons in the "on-shell" (Minkowskian) processes intensively discussed in the current literature (see discussion in ref. [4]

Finally, we note that our method gives same results as the conventional approach - namely position and strength of the renormalon singularity, but not the coefficient in front of it.

\footnotetext{
$\S \S$ The only difference would be that the upper limit for the integration over $t$ will be $\infty$ rather than 1 because the arbitrary valley starts at the perturbative vacuum and goes to the infinity with constantly increasing action .
} 
The coefficient in front of the first IR renormalon is expremely important since it determines the numerical value of asymptotics of perturbative series for $R_{e^{+} e^{-} \rightarrow \text { hadrons. In principle, for }}$ a given valley it is possible to calculate this coefficient in the first order in coupling constant $g^{2}(\rho)$ since it is given by a product of the determinants in the valley background, which can at least be computed numerically. However, the fact that an extra $g^{2}(\rho)$ does not change the position and/or character of the singularity means that all terms in the perturbative series in $g^{2}(\rho)$ contribute on equal footing. This is closely related to the fact that the choice of the valley is not unique: after the change of the valley, the new leading-order coefficient, which comes from the determinants in the background of a new configuration, is given by an infinite perturbative series in $g^{2}(\rho)$ (coming from quantum corrections) in terms of the original valley. As a result, finding the coefficient in front of the leading IR singularity would require the integration over all possible valleys. This study is in progress.

Acknowledgements. One of us (I.B.) is grateful to V.M. Braun, S.J. Brodsky, R.L. Jaffe, A.H. Mueller, and E.V. Shuryak for valuable discussions. This work was supported by the US Department of Energy under contracts DE-AC05-84ER40150 and DE-FG02-97ER41028.

\section{APPENDIX}

We will prove that rescaling of the arbitrary configuration by factor $\lambda$ (so that $\rho \rightarrow \lambda \rho$ ) leads at the one-loop level to multiplication of the determinant by a factor of

$$
\lambda^{b S / 8 \pi^{2}}
$$

where $S$ is the action of this configuration. Let us demonstrate it for the determinant of the Dirac operator in the background of a field $A_{\mu}(x ; \rho)$ characterized by the size $\rho$. We will prove that ${ }^{\text {*3*; }}$

\footnotetext{
*** For simplicity, we assume that the Dirac operator has no zero modes, as in the case of the $I \bar{I}$

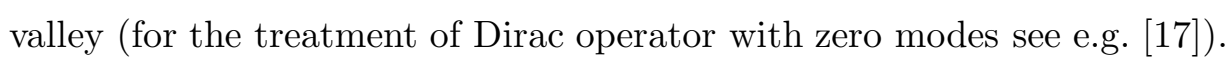




$$
\sqrt{\operatorname{det} \tilde{p}^{2}}=\sqrt{\operatorname{det} p^{2}} \lambda^{\frac{S}{12 \pi^{2}}}
$$

where $P_{\mu}=i \partial_{\mu}+A_{\mu}(x)$ is the operator of the covariant momentum for our configuration and $\tilde{P}_{\mu}=i \partial_{\mu}+\tilde{A}_{\mu}(x)$ is the covariant momentum in the background of the stretched configuration $\tilde{A}_{\mu}(x)=A_{\mu}(x ; \lambda \rho)=\lambda^{-1} A_{\mu}\left(\lambda^{-1} x ; \rho\right)$. Using Schwinger's notations we can write down

$$
\ln \operatorname{det} \tilde{P}^{2}=-\int_{0}^{\infty} \frac{d s}{s} \int d^{4} x \operatorname{Tr}\left(x\left|e^{-s \tilde{P}^{2}}\right| x\right)
$$

where $\mid x)$ are the eigenstates of the coordinate operator normalized according to $(x \mid y)=$

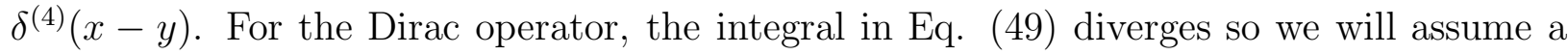
cutoff $s>\epsilon$ and take $\epsilon \rightarrow 0$ in the final results. After change of variables $x \rightarrow \lambda^{-1} x$, the integral in the r.h.s. of Eq. ( $\left(_{1} \overline{9} \bar{g}_{1}\right)$ reduces to

$$
\ln \operatorname{det} \tilde{p}^{2}=-\int_{0}^{\infty} \frac{d s}{s} \int d^{4} x \operatorname{Tr}\left(x\left|e^{-s \lambda^{-2} \not P^{2}}\right| x\right)
$$

so

$\ln \operatorname{det} \tilde{p}^{2}-\ln \operatorname{det} p^{2}=$

$$
\int_{0}^{\infty} \frac{d s}{s} \int d^{4} x \operatorname{Tr}\left(x\left|e^{-s \lambda^{-2} \not^{2}}-e^{-s \not^{2}}\right| x\right)=-\lim _{\epsilon \rightarrow 0} \int_{\lambda^{-2} \epsilon}^{\epsilon} \frac{d s}{s} \int d^{4} x \operatorname{Tr}\left(x\left|e^{-s P^{2}}\right| x\right)
$$

Using the well-known result for the DeWitt-Seeley coefficients for the Dirac operator (see e.g. [표묨]

$$
\operatorname{Tr}\left(x\left|e^{-s \not \not^{2}}\right| x\right)=\frac{3}{4 \pi^{2} s^{2}}+\frac{1}{48 \pi^{2}} G_{\mu \nu}^{a}(x) G_{\mu \nu}^{a}(x),
$$

we obtain

$$
\ln \operatorname{det} \tilde{P}^{2}=\ln \operatorname{det} P^{2}-\frac{\ln \lambda}{24 \pi^{2}} \int d^{4} x G_{\mu \nu}^{v a} G_{\mu \nu}^{v a}
$$

which corresponds to the Eq. ('i $(198)$. In the case of $n_{f}$ quark flavors, the coefficient $\frac{1}{12 \pi^{2}}$ in r.h.s.

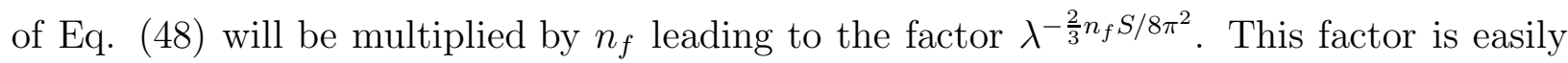
recognized as a quark part of the one-loop coefficient $b$ for Gell-Mann-Low $\beta$-function in Eq. (Ā 
gluon part of the one-loop coefficient $b$ in Eq. (푸국). Thus we have shown that $e^{-\frac{S}{g^{2}}} \rightarrow e^{-\frac{S}{g^{2}(\rho)}}$ with one-loop accuracy. Using the two-loop formulas for the Seeley coefficients one could prove that $e^{-\frac{S}{g^{2}(\rho)}}$ reproduces at the two-loop level and demonstrate that $g^{2} \rightarrow g^{2}(\rho)$ in the pre-exponential factor $g^{-17}$ as well (as it follows from the renormalizability of the theory).

[1] Large order behavior of perturbation theory, ed. J.C. Le Guillou and J. Zinn-Justin, NorthHolland, Amsterdam (1990).

[2] L.N. Lipatov, Sov. Phys. JETP 44 (1976) 1055; 45 (1977) 216.

[3] G 't Hooft, in The why's of Subnuclear Physics (Erice, 1977), ed A. Zichichi (Plenum, New York, 1977)

[4] M.Beneke, Phys. Reports 317 (1999) 1.

[5] E.B. Bogomolny and V.A. Fateyev, Phys. Lett. 71B (1977) 93.

[6] I. Balitsky and A.V. Yung, Phys. Lett. 168B (1986) 113; Nucl. Phys.B 274 (1986) 475

[7] M.Beneke and V.M.Braun, Nucl. Phys.B 426 (1994)301;

I.I. Bigi, M.A. Shifman, N.G. Uraltsev, and A.I. Vainshtein, Phys. Rev.D 50 (1994) 2234.

[8] A.V. Yung, Nucl. Phys.B 297 (1988) 47.

[9] I. Balitsky and A. Schafer, Nucl. Phys.B 404 (1993) 639.

[10] I. Balitsky, Phys. Lett. 273B (1991) 282.

[11] M.A. Shifman, A.I. Vainshtein, and V.I. Zakharov, Nucl. Phys.B 147 (1979) 1.

[12] A.H. Mueller, Nucl. Phys.B 250 (1985)327.

[13] M.S. Dubovikov and A.V. Smilga, Nucl. Phys.B 185 (1981)109, W. Hubschmid and S. Mallik, Nucl. Phys.B 207 (1982) 29. 
[14] M. Beneke, V.M.Braun, and N. Kivel, Phys. Lett. 457B (1999)147.

[15] M.N. Chernodub, M.I. Polikarpov, and V.I. Zakharov, Phys. Lett. 404B (1997)315.

[16] S.J. Brodsky, E. Gardi, G. Grunberg, and J. Rathsman, Conformal expansion and renormalons, SLAC-PUB-8362, CPTH-S-746-1099, LPT-ORSAY-00-09, CERN-TH-2000-032, Feb 2000. e-Print Archive:

[17] A.S. Schwarz, Quantum field theory and topology, Springer Verlag, 1993.

[18] B.S. DeWitt, Dynamical theory of groups and fields, Gordon and Breach, New York, 1965 